\title{
A Framework for Process Reengineering in Higher Education: A case study of distance learning exam scheduling and distribution
}

\author{
M'hammed Abdous and Wu $\mathrm{He}$ \\ Old Dominion University, USA
}

\begin{abstract}
In this paper, we propose a conceptual and operational framework for process reengineering (PR) in higher education (HE) institutions. Using a case study aimed at streamlining exam scheduling and distribution in a distance learning (DL) unit, we outline a sequential and non-linear four-step framework designed to reengineer processes. The first two steps of this framework - initiating and analyzing - are used to initiate, document, and flowchart the process targeted for reengineering, and the last two steps - reengineering/ implementing and evaluating - are intended to prototype, implement, and evaluate the reengineered process. Our early involvement of all stakeholders, and our in-depth analysis and documentation of the existing process, allowed us to avoid the traditional pitfalls associated with business process reengineering (BPR). Consequently, the outcome of our case study indicates a streamlined and efficient process with a higher faculty satisfaction at substantial cost reduction.
\end{abstract}

Keywords: process reengineering; exam scheduling and distribution; distance learning.

\section{Introduction}

Business process reengineering (BPR) is the analysis and design of workflows and processes within and between organizations (Davenport \& Short, 1990). In spite of academics' often skeptical stance against BPR and other management concepts (Birnbaum, 1988), which may seem foreign to the organizational culture of higher education (HE), many universities have reengineered their internal processes. According to a recent study conducted by Educause (Kvavik, Goldstein, \& Voloudakis, 2005), HE institutions have invested heavily in business process reengineering, leveraging information technology to improve services and reduce costs. This interest in rethinking processes and procedures is driven mainly by budget shortfalls, information technology infusion, and external pressures for greater accountability and responsiveness. Despite this enthusiasm and heavy investment from HE institutions however, few studies have examined the overall effectiveness and outcomes of reengineered processes. Additionally, with the exception of the above mentioned Educause study, Allen and Fifield (1999), and the work of Sepehri, Mashayekhi and Mozaffar (2004), Okunoye, Frolick and Crable (2006), and to some extent Penrod and Dolence (1992) and Belarmino and Canteli (2001), few studies have proposed a comprehensive framework to reengineer processes in HE environment. In this paper, we attempt to contribute to both aspects by proposing a conceptual and operational 
Abdous \& He

framework for process reengineering in a HE environment, and by examining its effectiveness through a case study from a DL unit. This case study had two specific goals: (1) to replace a cumbersome and inefficient paper-based exam distribution and scheduling process with a Webbased streamlined and efficient process; and (2) to reduce the overall cost associated with mailing exams to/ from remote DL sites.

\section{Process Reengineering Framework}

In their seminal work on BPR, Hammer and Champy (1993) are credited in the literature as defining reengineering as "the fundamental rethinking and radical redesign of business processes to achieve dramatic improvements in critical temporary measures of performance such as cost, service, quality, and speed" (p. 46). Closer to the organizational culture of HE, and prior to the Hammer and Champy work, Penrod and Dolence (1992) defined reengineering as "using the power of modern information technology to radically redesign administrative business processes in order to achieve dramatic improvements in their performance” (p. 8). From these two definitions, we understand that the ultimate goal of process reengineering is to achieve efficiency and effectiveness by radically rethinking existing processes; whereas the goal of total quality management is to undertake process change gradually by working in incremental steps (O’Neill \& Sohal, 1999).

One of the corollary outcomes of BPR's pursuit of efficiency and effectiveness often is translated into staff reduction and downsizing. By injecting new ways of doing things, BPR provides the opportunity to senior leadership to reduce staff and to reshape the organizational culture, as reflected in its values, norms, guidelines, and expectations, (Schein, 2004).

Following the BPR perspective, we designed a framework inspired by two main sources: (1) a retrospective analysis of our own experience in reengineering several internal processes, such as faculty development program management (Abdous, 2005), a syllabus creation process (Abdous \& He, 2006), and learning assessment lab registration; and (2) the BPR literature (Davenport \& Short, 1990; Macintosh, 2003, O’Neill \& Sohal, 1999; Ahmad, Francis \& Zairi, 2007). By combining two sources in which theory has been nurtured by practice, our framework provides a well grounded tool to use when reengineering processes in HE. As shown in the following figure, our framework is structured around four sequential and non-linear phases. 
Abdous \& He

Figure 1. Proposed process reengineering framework

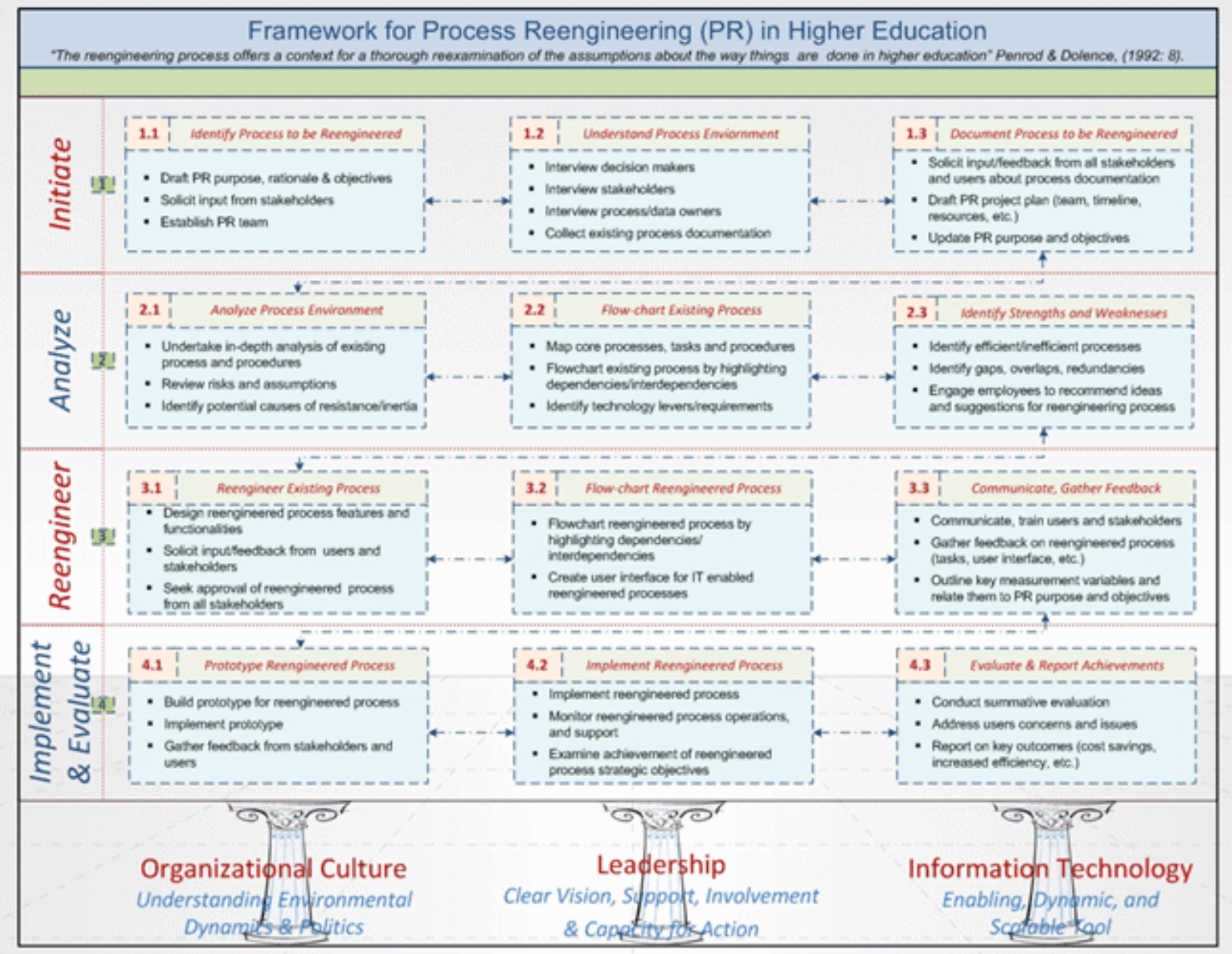

These four sequential and non-linear phases are:

- Initiation. This phase is aimed at identifying and understanding the purpose, the rationale, and the objectives of the process review (PR). This phase requires active participation from all stakeholders to document and to understand the environmental dynamics and the risks associated with the proposed reengineering process (den Hengst \& de Vreede, 2004).

- Analysis. This second phase involves an in-depth analysis of the process tasks and procedures by analyzing tasks, reviewing risks and assumptions, and by identifying potential causes of resistance and inertia. With the documentation obtained during phase one, the outcome of this analysis phase is used to flowchart the process. A visual presentation of the existing process dependencies and interdependencies is not only critical to mapping the core tasks and procedures of the process, but is also foundational for the reengineering process itself. 
Abdous \& He

- Reengineering. This third phase is intended to design the features and functionalities of the reengineered process, and includes active participation and feedback from all appropriate personnel and users. As the process is redesigned, the flowchart outlined in phase two is updated to include a clarification of the key measurement variables. In this phase, information technology is used as an enabling and facilitating tool.

- Implementation and evaluation. The implementation and evaluation phase is intended as a first step to prototype the reengineered process, thus addressing users' and stakeholders' issues and concerns. In a second step, the reengineered process is fully implemented and monitored to ensure successful operation. A summative evaluation is conducted, and achievement and outcomes are reported.

Successful implementation of this framework requires three key pillars: (1) a deep understanding and familiarity with the organizational culture and its dynamics and politics (den Hengst \& de Vreede, 2004), (2) a clear vision, involvement, and support from senior leadership (O’Neill \& Sohal, 1999) sustained by a "capacity for action" (Greenwood \& Hining, 1996), and (3) the intelligent leveraging of information technology as an enabling, dynamic, and scalable tool (Ahmad, Francis \& Zairi, 2007; Kohli \& Hoadley, 2006).

\section{Process Reengineering Framework Application}

Our case study was conducted at a moderately sized, urban, public, doctorate awarding, research university. Despite the fact that, in recent years, the university's delivery modes have been expanded to include two-way video, Internet, CD-ROM, and synchronous video streaming, the majority of its distance courses still are delivered using interactive television. Between Summer 2006 and Summer 2007, 593 instructors taught 1,281 courses, totaling 37,668 registrations. With numbers this high, material distribution and paper-based exam scheduling logistics can be challenging and sometimes overwhelming (Abdous \& He, 2007). Indeed, in addition to burdening faculty with numerous paper-based forms for each exam given, this manual approach created tracking, processing, and retrieving problems for the DL staff. In short, the submission of paper forms was costly, cumbersome, and inefficient, and made tracking and managing difficult. To address these issues, a Web-based solution was designed, following the four step framework outlined above.

\section{1) Initiation}

In this phase, we identified the exam scheduling and distribution process to be reengineered from the perspective of our unit's years of experience in dealing with exam scheduling and distribution for DL courses' material and exams. We established our understanding of the process by reviewing a variety of sources - including original paper reports, manuals, and forms - and by interviewing current staff and administrators in DL and, perhaps more importantly, faculty members teaching DL courses. Although nowadays a large number of objective exams are conducted using course management systems such as Moodle, the majority of televised courses exams, essays, and papers still are conducted and managed traditionally in a classroom environment using paper and pencil. 
Abdous \& $\mathrm{He}$

After observing the exam scheduling and distribution processes used in our DL unit, we conducted a series of meetings with related stakeholders in order to cultivate a supportive environment, get their feedback, and document the process to be reengineered. During multiple meetings, we established a development team; drafted a project plan; and defined team roles, accountability, expectations, and timelines in order to facilitate project management.

\section{2) Analysis}

In this step, we conducted an in-depth analysis of the tasks and procedures involved in the exam scheduling and distribution process. In-depth evaluations and analysis of the current paper-based exam scheduling and distribution approach were made and artifacts were collected. As a result, we flowcharted the existing process (see Figure 2) and identified a list of issues and problems which needed to be addressed. The team met many times, reached an agreement, and made recommendations for the reengineered process which included new features, functionalities, requirements, and ideas.

Figure 2. Paper-based exam distribution and scheduling process

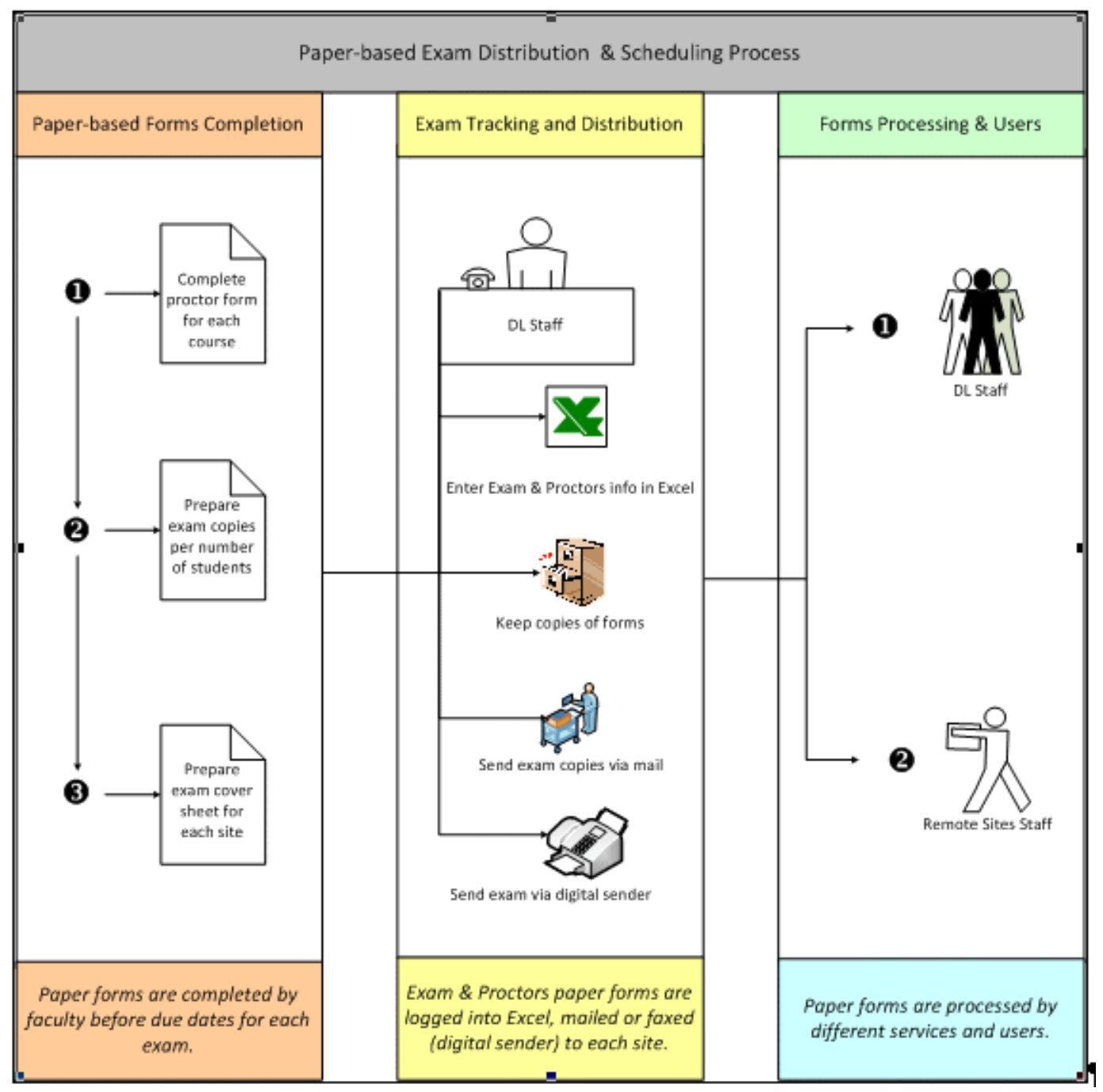


Abdous \& He

\section{3) Reengineering}

In this step, we designed the features and functionalities of the reengineered process. Specifically, we flowcharted the reengineered process and conducted a system conceptual design and an interface design. We developed a conceptual model of the phases, tasks, and functions, and then created a "database-related entity-relationship" diagram (see Figure 3). Subsequently, we formalized this conceptual design by creating a Web-based user interface involving all system users. This participative approach during the design phase enabled us to capture users' concerns and feedback early in the process, and to reach a consensus as to the workability of the reengineered process.

Figure 3. Web-based exam distribution and scheduling process

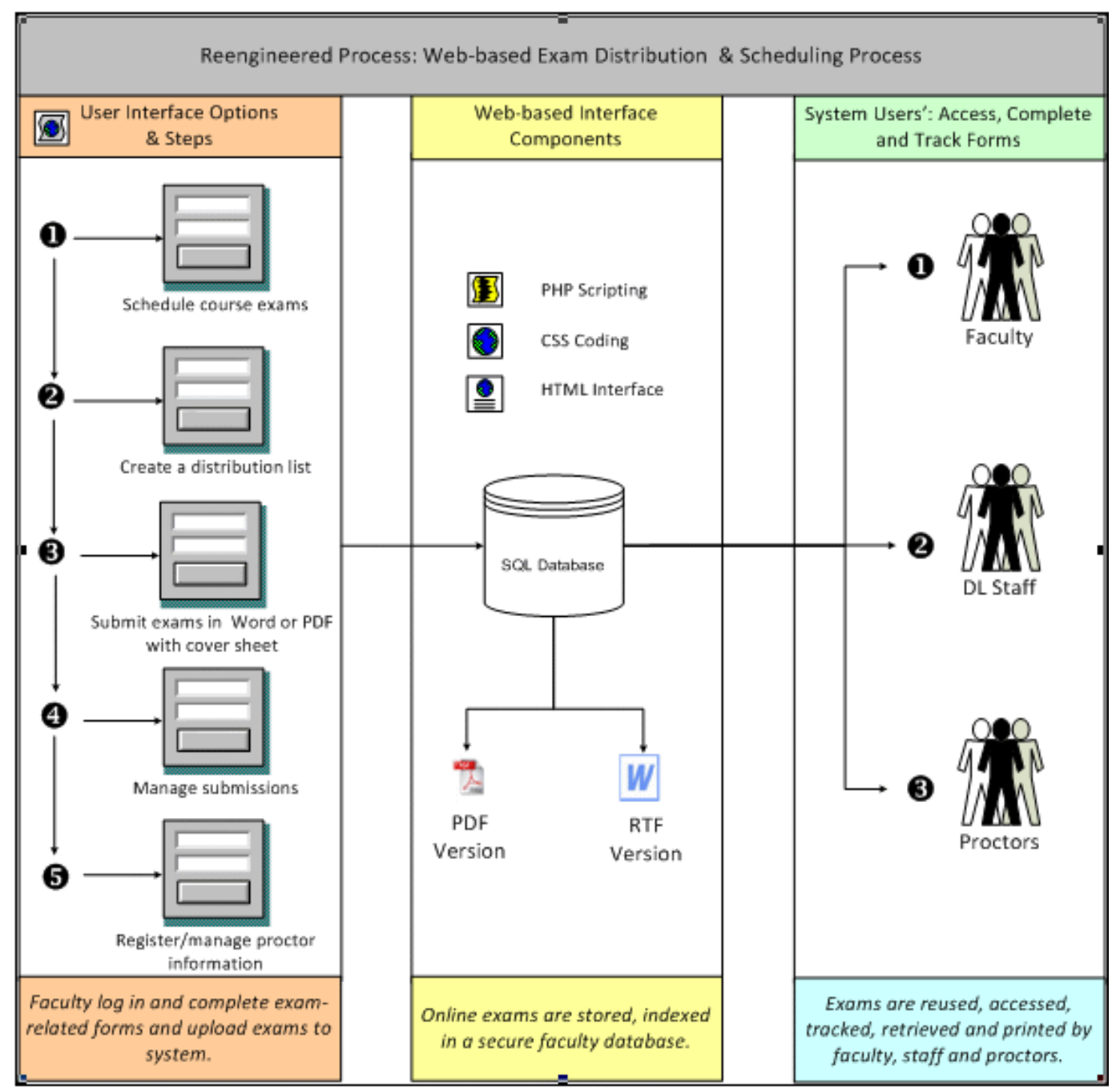

During the reengineering process, an instructional designer, two instructional technologists, and an interface designer were involved in the actual system programming and development. The system is a database driven environment intended to streamline the exam related forms 
Abdous \& He

management process. To deploy the system on the Web, a Microsoft MSSQL server was used as the backend database environment. It was chosen because of its rich capabilities in supporting the required functionalities. PHP was used as the scripting language in order to create dynamic Web content by querying the database. Cascading Style Sheets (CSS), a common style sheet language for webpages, was used to ensure the overall consistency of the system's look and feel. A user account authentication system maintained by the Center for Learning Technologies was used to prevent unauthorized access to the online form services, thereby enforcing the security of the application.

\section{Reengineered Process Features and Steps}

The reengineered process allows for access by three types of users: faculty, DL support staff, and proctors (people appointed to supervise students at an examination). On the faculty accessed portion of the site (see Figure 4), faculty members are able to accomplish the following tasks/ steps:

Step 1: Schedule course exams. Faculty members schedule all of their exams for the entire semester and indicate whether or not they will need a proctor.

Step 2: Create a distribution list. Faculty members create their course's distribution list, which is used to send/ return exams or course related materials to the remote sites. This list also is used to notify remote sites' staff of any schedule changes. It is worth mentioning at this juncture, that before this process was reengineered, the list was entered manually for each site. With the reengineered process, the system fills in the data using an automated report process from the university data system.

Step 3: Submit exams. Faculty members upload their exam documents in Word or PDF format. To maintain cross platform consistency, Word documents are converted to PDF format. Using a generated distribution list, instructions, cover sheets, and exams are emailed to the remote sites' staffs, as well as to faculty and proctors.

Step 4: Manage submissions. Faculty members are able to modify and/ or update exam information and schedule or update distribution lists. DL staff and proctors are notified of any changes.

On the staff side, DL support staff members are able to track the course exams and material by course name and date, and can monitor and respond to course and exam modification requests from faculty. Staff members also can submit course and exam modification requests to remote sites on behalf of faculty members, as needed. 
Abdous \& He

Figure 4. A Web-based exam distribution and scheduling process interface

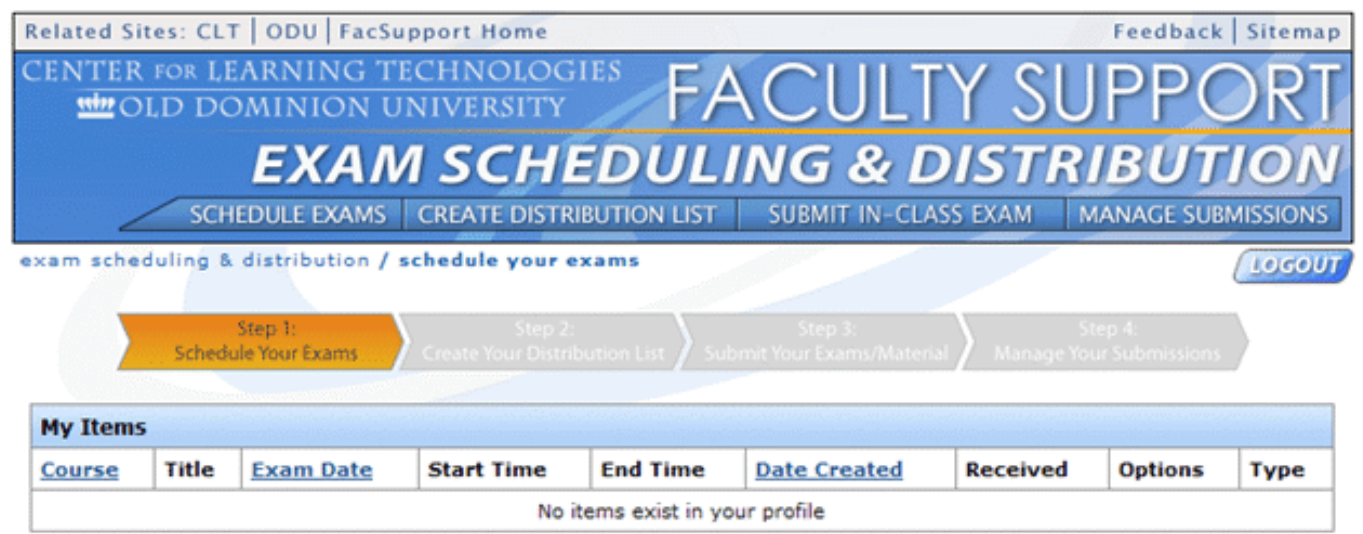

On the proctor page, a request form for DL students and a proctor agreement form also are available online. DL support staff members are able to track and approve the proctor request and agreement information.

\section{4) Implementation and evaluation}

The development team adopted a rapid application development approach (Robinson, 1995) in building the system. Based on the entity relationship diagrammed in Step 3, we designed a database using a Microsoft SQL server, followed by a shared login/ authentication module. After that, we created a separate module including access privileges and features for each user. These modules then were integrated into a fully functional system. The user features and functions were prototyped and tested with real data several times prior to the actual use of the system. The system (see http://www.clt.odu.edu/mdf/) has now been through multiple iterations of revision, based on feedback collected from all users.

\section{Evaluation of the Online Exam Scheduling and Distribution System}

The online system has been up and running since the fall semester of 2006. To capture firsthand faculty feedback and to explore their overall satisfaction with the usability of the system, a Webbased survey was administered to all faculty members using the system. The intent of the survey was twofold: (1) to understand how faculty members were using the system; and (2) to examine the impact of the system on reducing faculty workload. The collected data is also expected to inform future development and research on the system.

A quick analysis of the data indicates that 32 faculty members representing various disciplines and colleges (out of 150 users) completed the survey. The instrument contained five items that could be responded to on a 5-point Likert-type scale (see Table 1) and also contained an open ended question asking participants to recommend specific system improvements. Descriptive statistics were used to analyze the rating scale and a content analysis of faculty answers was conducted to catalog the open ended questions. 
Abdous \& He

Table 1. Overall faculty satisfaction with the exam scheduling system

\begin{tabular}{|l|c|c|c|c|c|}
\hline Dimensions & $\begin{array}{c}\text { Strongly } \\
\text { agree }\end{array}$ & $\begin{array}{c}\text { Modenteb } \\
\text { Agree }\end{array}$ & Neutral & $\begin{array}{c}\text { Modenteb } \\
\text { Disagne }\end{array}$ & $\begin{array}{c}\text { Strongly } \\
\text { Disagree }\end{array}$ \\
\hline $\begin{array}{l}\text { Has all of the functions and } \\
\text { capabilities I expect-it to have }\end{array}$ & $21 \%$ & $33 \%$ & $15 \%$ & $18 \%$ & $6 \%$ \\
\hline $\begin{array}{l}\text { Enables me to accomplish tasks } \\
\text { more quickly }\end{array}$ & $30 \%$ & $24 \%$ & $12 \%$ & $18 \%$ & $9 \%$ \\
\hline Easy to use & $12 \%$ & $39 \%$ & $18 \%$ & $21 \%$ & $6 \%$ \\
\hline $\begin{array}{l}\text { Reduces-my time and effort in } \\
\text { managing exam schedules and } \\
\text { distribution to DL students }\end{array}$ & $18 \%$ & $24 \%$ & $21 \%$ & $12 \%$ & $15 \%$ \\
\hline $\begin{array}{l}\text { Makes it easier to meet } \\
\text { submission deadlines }\end{array}$ & $27 \%$ & $33 \%$ & $15 \%$ & $6 \%$ & $12 \%$ \\
\hline & & & & & \\
\hline
\end{tabular}

Using a 5-point scale ranging from 1(low) to 5 (high), participants rated their comfort levels about using computers and the Web. Their average comfort level was 4.06 with computers and 4.37 with the Web. Overall, approximately 51 percent of the participating faculty members indicated that the system was easy to use. Their feedback indicates that the system (1) enables them to accomplish tasks more quickly (54\%); (2) reduces time and effort in managing exam schedules and in distribution to DL students (42\%); and (3) makes it easier to meet submission deadlines (60\%). The results presented in Table 1 suggest that many faculty members have a positive attitude about the system. As the system remains an ongoing process and is not integrated with other university systems, such as the university registration system, faculty members must create a new account to access the system. To some degree, the account issue has contributed to the dissatisfaction of some faculty with the system.

The survey results also reveal some difficulties in the faculty's use of the system, as well as some opportunities for the DL staff to improve the system. Approximately 43.75 percent of the faculty members participating in the survey indicated that the system requires more functions and capabilities. Approximately 65.63 percent indicated that they needed assistance in using the system. Some faculty recommendations include:

- Include a short video on how to use the system.

- Integrate with other university systems.

- Convert uploaded Word files automatically into PDF files for exams to avoid version inconsistency.

- Email the receipts from the various sites to the faculty after the exam submission,

- Email reminders to faculty members who do not submit the exam as scheduled.

In response to these recommendations, we purchased software to automatically convert Word files into PDF files on the server side (without human intervention). In addition, the system now sends an automatic email receipt to faculty after each exam submission. In the same way, the 
Abdous \& He

system reminds faculty members of their schedule deadlines. An audio/ video tutorial is planned for implementation later this year, after our second round of evaluations.

Although cost/ benefit analysis is traditionally required to demonstrate cost savings in BPR projects, we decided to report cautiously that our project generated direct and indirect cost savings. Our unit accounting office calculated the annualized cost saving attributed to the reengineered system at US $\$ 7,500$, which accounts for eight percent of the mailing budget. This estimate included direct costs associated with mailing charges only. We expect this percentage to increase once all DL faculty members are using the system. In indirect cost saving, the new functionalities of the system freed faculty and staff from clerical and time consuming tasks. We assume that this workload reduction likely will contribute to increased faculty and staff productivity.

In brief, despite a slight resistance from staff, which is typical of BPR implementation building around any legacy system, we believe that we achieved our stated goals of: (1) replacing a cumbersome and inefficient paper-based exam distribution and scheduling process with a Webbased streamlined and efficient process; and (2) reducing both the faculty/ staff workload associated with the clerical tasks of scheduling exams and the overall costs associated with exam distribution.

\section{Conclusion and Future Work}

In this paper, we have presented an online exam scheduling and distribution system for DL courses. The system plays an essential role in facilitating coordination and communication among the stakeholders involved in the exam scheduling and distribution process. Further improvements are planned to integrate the system with university registrar data systems. This integration will increase automated tasks and reduce faculty need to input student and course data. In addition, we are examining how to help faculty manage graded exam and paper collection and distribution.

In summary, drawing from BPR research and from our own experience in redesigning processes, our proposed framework offers a flexible roadmap to rethink, redesign, and streamline the exam scheduling processes. Our operational model is rooted in the organizational culture of HE, and actively engages all stakeholders to undertake an in-depth analysis and take ownership of the reengineering process. Our application of the framework has enabled us to uncover inefficiencies, reduce paper handling, increase efficiency, and achieve operational improvements while reducing faculty workload and reducing overall cost. We believe that our framework will be used to identify and streamline other services and will be of assistance to other universities' DL efforts.

\section{Acknowledgments}

We want to thank the staff in the Center for Learning Technologies for their help in designing the system. We also wish to thank the DL faculty at Old Dominion University for their assistance in the design and the development of the system.

\section{References}

Abdous, M. (2005). Designing a faculty development portal and architecture. In Proceedings of the 21st Annual Conference on Distance Teaching and Learning, 2005. 
Abdous \& He

Madison, WI.

http://www.uwex.edu/disted/conference/Resource_library/proceedings/05_1966.pdf

Abdous, M., \& He, W. (2006). A learner-centered syllabus generator system. In Proceedings of the International Conference on Society for Information Technology \& Teacher Education, Orlando, FL, March, 2006.

http://www.editlib.org/index.cfm?fuseaction=Reader.PrintAbstract\&paper_id=22198

Abdous, M., \& He, W. (2007). Streamlining Forms Management Process in a Distance Learning Unit. Journal of Distance Learning Administration, 10(2). http://www.westga.edu/ distance/ojdla/summer102/abdous102.htm

Ahmad, H., Francis, A., \& Zairi, M. (2007). Business process reengineering: critical success factors in higher education. Business Process Management Journal, 13(3), 451-469.

Allen, D., \& Fifield, N. (1999). Re-engineering change in higher education. Information Research, 4(3). http://informationr.net/ir/4-3/paper56.html

Belarmino, A., \& Canteli, A. (2001). Business process reengineering and university organisation: A normative approach from the Spanish case. Journal of Higher Education Policy and Management, 23(1), 63-73.

Birnbaum, R. (1988). How colleges work: The cybernetics of academic organizations and leadership. San Francisco: Jossey-Bass.

Davenport, H., \& Short, E. (1990). The new industrial engineering: Information technology and business process redesign. Sloan Management Review 31(4),11-27.

den Hengst, M., \& de Vreede, G. (2004). Collaborative business engineering: A decade of lessons from the field. Journal of Management Information Systems, 20(4), 85-114.

Greenwood, R., \& Hinings, R. (1996). Understanding radical organizational change: Bringing together the old and the new institutionalism. Academy of Management Review, 21(4), 1022-1054.

Hammer, M., \& Champy, J. (1993). Re-engineering the corporation, a manifesto for business revolution. New York: Harper Collins.

Kohli, R., \& Hoadley, E. (2006). Towards developing a framework for measuring organizational impact of IT-enabled BPR: case studies of three firms. SIGMIS Database 37(1), 40-58. http://portal.acm.org/citation.cfm?id=1120505

Kvavik, R. B., Goldstein, P., \& Voloudakis, J. (2005). Good enough! IT investment and business process performance in higher education Educause. http://connect.educause.edu/Library/ECAR/GoodEnoughITInvestmentand/41156?time=1 203515704

Macintosh, R. (2003). BPR: Alive and well in the public sector. International Journal of Operations \& Production Management, 23(3), 327-344. 
Abdous \& He

Okunoye, A., Frolick, M., \& Crable, E. (2006). ERP implementation in higher education: An account of pre-implementation and implementation phases. Journal of Cases on Information Technology, 8(2), 110-132.

O’Neill, P., \& Sohal, A. (1999). Business Process Reengineering. A review of recent literature. Technovation, 19(9), 571-581.

Penrod, J., \& Dolence, M. (1992). Reengineering: A process for transforming higher education. Proceedings of the 21st Annual Conference on Distance Teaching and Learning 2005. Boulder CO: CAUSE, Professional Paper Series, \#9.

Robinson, R. (1995). Radical systems development: An introduction to rapid application development. Chapel Hill, NC: Professional Press.

Schein, E. (2004). Organizational culture and leadership (3rd ed.). New York: Wiley.

Sepehri, M., Mashayekhi, A. \& Mozaffar, A. (2004). Transformation of higher education system in a developing country: Case of decentralization and reengineering of faculty hiring process. UNESCO Portal. http://portal.unesco.org/education/en/ev.php-

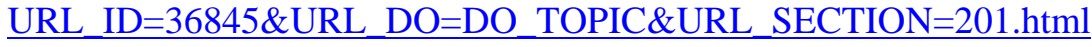

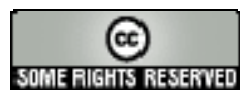

